\section{La stérilisation des malades et handicapés mentaux: hier et aujourd'hui}

La question de la stérilisation des malades et handicapés mentaux est revenue récemment en force $\dot{a}$ l'avant-plan de l'actualité helvétique, dans le contexte général du réexamen de l'histoire de la Suisse pendant la première moitié du XXe siècle. A-t-on stérilisé des malades mentaux? Si oui, à quelle échelle? Et quel a pu être le lien de cette pratique avec le contexte politique et idéologique qui a mené aux tragédies que l'on sait? Notons d'emblée que le réexamen de certaines pratiques impliquant la médecine et dirigées spécifiquement à l'encontre de certains groupes sociaux n'est pas propre à notre pays, et qu'il est, ici comme ailleurs, vif et passionné.

Les deux études présentées ci-après se penchent sur l'histoire de la stérilisation des malades mentaux dans deux cantons différents, Argovie et Vaud, dont les pratiques en la matière apparaissent distinctes sur certains points. Tout d'abord, dans le canton de Vaud existe dès 1928 une loi sur la stérilisation des infirmes et malades mentaux (elle fut en son temps considérée internationalement comme pionnière), alors que le canton d'Argovie, comme d'ailleurs le reste de la Suisse, fonde sa pratique en la matière sur une sorte de consensus qui n'est pas fixé par des réglementations précises. Cette simple différence de contexte légal induit des possibilités d'interprétation multiples, qui illustrent bien l'illusion consistant $\dot{a}$ exiger du travail de l'historien - sur ce thème comme sur d'autres - qu'il restitue une vérité historique univoque. La question de l'implication du "malade», notamment, est particulièrement délicate: Mme Dubach souligne qu'un tel consentement était exigé dans le canton d'Argovie, mais que dans bien des cas, il dut faire l'objet de pressions de l'entourage. Dans le canton de Vaud en revanche, la loi n'exigeait pas un tel consentement; or, Mr Jeanmonod et al. montrent comment cette loi, qui peut d'emblée paraître comme une mesure coercitive, put également avoir un effet protecteur, en permettant dans certains cas de réfréner une pratique que certains auraient voulu voir appliquer avec plus de libéralité.

Les deux articles mettent par ailleurs en relief des caractéristiques communes particulièrement frappantes: dans un canton comme dans l'autre, ce furent essentiellement, dès les années 1920 et jusque tard dans le siècle, des femmes de condition sociale souvent défavorisée qui furent l'objet d'une mesure de stérilisation. Autre trait commun, les motifs qui menèrent à cette intervention furent rarement d'ordre eugénique pur. Comme ces études historiques le soulignent, il importe en effet, tout en en reconnaissant les liens, de distinguer l'eugénisme à proprement parler, et l'appareil technocratique et policier qui lui est lié, des mesures d'hygiène sociale, visant notamment à lutter contre les défauts (ou ce qui était ressenti comme tel) d'ordre social, moral et sanitaire.

De nombreux points dans l'une et l'autre étude appellent la comparaison avec la situation actuelle, et sont susceptibles de fournir quelques éléments de réflexion dans les débats éthiques contemporains. La question du consentement évoque inévitablement la pratique actuelle $d u$ consentement $d u$ patient dans des circonstances semblables. On peut aussi réfléchir sur la fragilité et la relativité des notions scientifiques qui, à un moment donné, apparurent comme parfaitement solides et capables de consolider, voire justifier, une politique impliquant des interventions d'une portée non négligeable sur les humains: que penser aujourd'hui des diagnostics d'alors comme la "faiblesse morale", et de notions associées, comme l'hérédité ou l'incurabilité de telles "maladies" mentales? Une histoire de la médecine bien menée consiste précisément à signaler tant ce qui nous rapproche que ce qui nous sépare des situations $d u$ passé. C'est ainsi qu'elle contribue à enrichir les débats cruciaux de la médecine d'aujourd'hui.

Prof. Dr Vincent Barras, Institut romand d'histoire de la médecine et de la santé, Lausanne 